\title{
Recurrent Uterine Corpus Sarcoma
}

National Cancer Institute

\section{Source}

National Cancer Institute. Recurrent Uterine Corpus Sarcoma. NCI Thesaurus. Code C8261.

The reemergence of uterine corpus sarcoma after a period of remission. 